\title{
Transmission Risks of Mountain-Type Zoonotic Visceral Leishmaniasis - Six Endemic Provincial-Level Administrative Divisions, China, 2015-2020
}

\author{
Xinyi Wang'; Shang $\mathrm{Xia}^{1,2}$; Jingbo Xue ${ }^{1,2} ;$ Zhengbin Zhou ${ }^{1}$; Yuanyuan $\mathrm{Li}^{1}$; \\ Zelin Zhu'; Yi Zhang ${ }^{1,2}$; Qiang Wang'; Shizhu Li ${ }^{1,2, *}$
}

\section{Summary}

What is already known about this topic?

Mountain-type zoonotic visceral leishmaniasis (MTZVL) cases in China have increased significantly between 2015 and 2020. A total of 25 regions had reemerged yielding 88 MT-ZVL indigenous cases, while the total number of visceral leishmaniasis cases declined.

\section{What is added by this report?}

The transmission risk of MT-ZVL showed a trend of patchy dissemination centered on major endemic areas and medium-high risk occurrence areas of Phlebotomus chinensis with discrete foci. Multi-point re-emergence and local outbreaks of MT-ZVL were trending in historically endemic areas.

What are the implications for public health practice?

Risk identification and early warnings of MT-ZVL are essential in formulating precise prevention and control strategies in China. More frequent monitoring, establishing a mechanism of joint prevention and control, and highlighting health education are recommended.

After large-scale control and treatment efforts, visceral leishmaniasis (VL) was well controlled in China by the 1960s and eliminated in the early 1980s as a major public health problem (1). However, the number of mountain-type zoonotic visceral leishmaniasis (MT-ZVL) cases has been increasing significantly in recent years. Conducting timely identification of this disease and implementing an early warning of MT-ZVL transmission risks are fundamental premises of generating targeted intervention measures. To identify the key risk areas, a risk-matrix was used to assess the transmission risk of MT-ZVL in six endemic provincial-level administrative divisions (PLADs) in China. Data such as the indigenous cases (2015-2020) and the occurrence probabilities of the main vector Phlebotomus chinensis (Ph. chinensis) were collected, and the comprehensive scores were calculated. The transmission risk areas showed a trend of patchy dissemination centered on major endemic areas and medium-high risk occurrence areas of the vector with discrete foci. Multi-point re-emergence and local outbreaks of MT-ZVL appeared in historically endemic areas. In the medium-high risk areas identified, the recommended strategies are more frequent monitoring of sandflies, canines, and mobile population, establishing a mechanism of joint prevention and control, and highlighting health education to the public.

The indigenous cases reported in 6 endemic PLADs were collected from the National Notifiable Disease Reporting System between 2015 and 2020 (Figure 1A). The data were verified by epidemiological investigations on individual cases and vector investigations conducted by county-level CDCs. According to the working guidelines of VL prevention and control, the endemic county-level administrative divisions (CLADs) were categorized into three classes with the number of reported cases based on experts' experiences of onsite prevention and control. Class I CLADs were those with a cumulative case number of not less than 10. Class II and Class III CLADs had 3-9 and less than 3 cases, respectively. Non-endemic CLADs were those that have no indigenous case. The scores were 3, 2, 1, 0, from more cases to less.

The historical distribution of the vector, bioclimatic, and geographical environmental data were collected. MaxEnt software (version 3.4.1, American Museum of Natural History, New York, NY, USA) was used to calculate the occurrence probabilities of $\mathrm{Ph}$. chinensis in a previous study (2). The continuous value of occurrence probabilities ranged from $0-1$ (minimal-maximal probability). In this study, the 

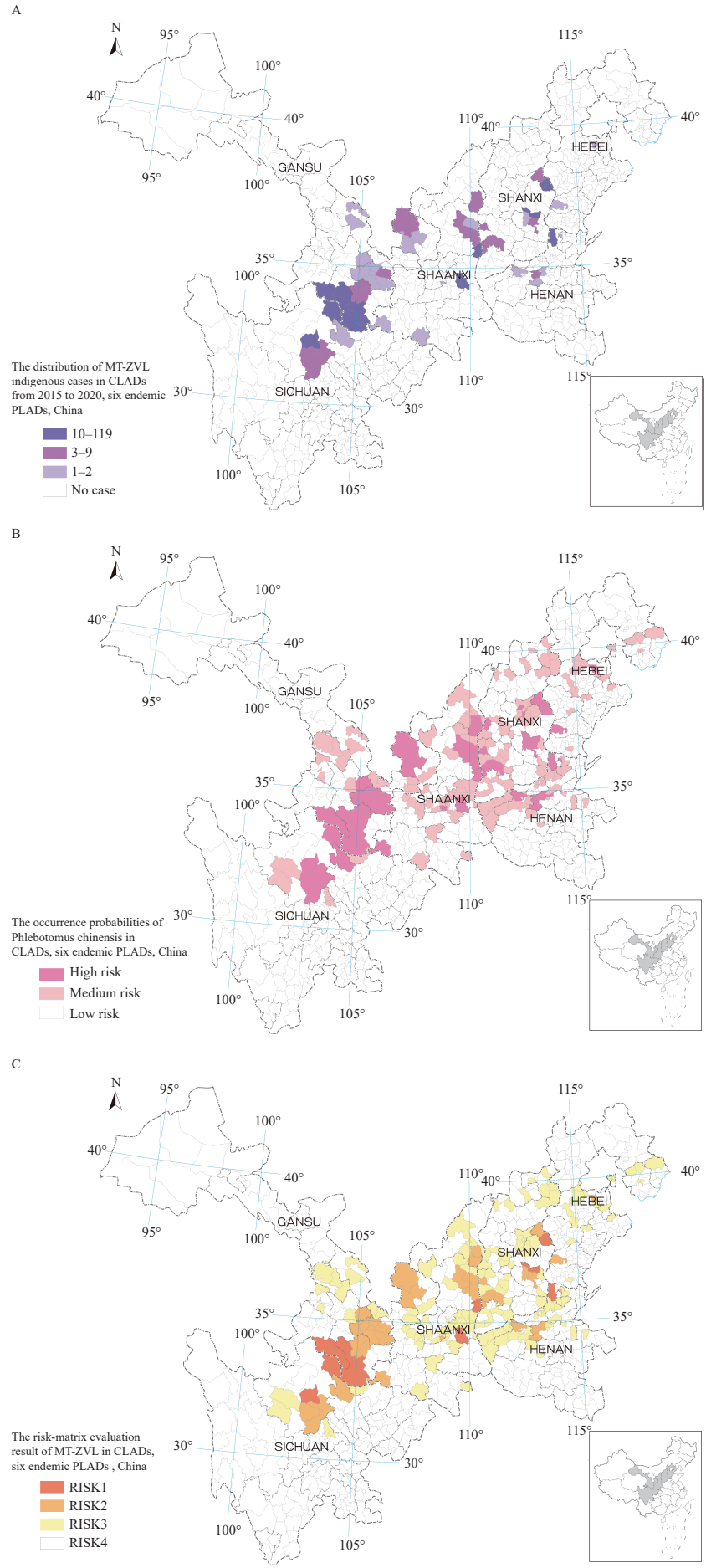

FIGURE 1. Transmission risks of mountain-type zoonotic visceral leishmaniasis in six endemic PLADs, China. (A) The distribution of MT-ZVL indigenous cases in CLADs from 2015 to 2020; (B) The occurrence probabilities of Phlebotomus chinensis in CLADs; (C) The risk-matrix evaluation result of MT-ZVL in CLADs.

Note: The occurrence probabilities of Phlebotomus chinensis were calculated using the distribution data of the vector, and bioclimatic and geographical environment data in previous studies. The sandfly distribution data was derived from a national field survey and historical literature; the environment data was derived from the WorldClim database (https://www.worldclim.org/data/worldclim21.html) and Resource and Environment Science and Data Center database (http://www.resdc.cn/).

Abbreviations: MT-ZVL=mountain-type zoonotic visceral leishmaniasis; PLADs=provincial-level administrative divisions; CLADs=county-level administrative divisions. 
occurrence probabilities were re-extracted by mean value based on CLADs and further categorized into three ranks. A $0.60-1.0$ value was a high risk for the occurrence of $\mathrm{Ph}$. chinensis, $0.30-0.59$ was medium risk, and $0-0.29$ was low risk, and these were assigned 3,2 , and 1 points, respectively.

The cumulative value of the comprehensive scores was calculated and presented in the transmission riskmatrix (Table 1). The assessment was under expert guidance and the results were already included in the investigation of vectors in potential MT-ZVL endemic areas. The data of indigenous cases and the vectors, and the risk warning assessment scores were analyzed by Microsoft Excel (version 2016, Microsoft, Redmond, WA, USA). The visual maps were made by ArcGIS (version 10.1, ESRI, Redlands, CA, USA).

The number of MT-ZVL indigenous cases and its proportion of total VL cases increased significantly between 2015 and 2020, although the annual number of VL cases showed an overall downward trend. Reemerged cases were defined as indigenous cases after epidemiological investigations that currently reoccurred in an area where local transmission was interrupted in the past 10 years, according to the literature (3). A total of $25 \mathrm{MT}-\mathrm{ZVL}$ re-emergent CLADs appeared between 2015 and 2020, with 88 reemergent cases reported. The number of class I, II , and $\amalg$ CLADs were 16, 20, and 23 in the study area, respectively. They were scattered in southern Gansu, northern Sichuan, and the mountainous cross-border areas of Shaanxi, Shanxi, Hebei, and Henan, showing significant local aggregation characteristic (Figure 1A). The occurrence probabilities map of $\mathrm{Ph}$. chinensis showed 66 high risk areas, 154 medium risk areas, and 592 low risk areas on a county basis (Figure 1B).

According to the risk assessment results, RISK 1, RISK 2, RISK 3, and RISK 4 areas contained 16, 41, 143 , and 592 CLADs, corresponding to super-high risk, high risk, moderate risk, and low risk, respectively. RISK 1 and RISK 2 areas included all major MT-ZVL endemic CLADs and the majority of high-risk areas for $P h$. chinensis occurrence, while most of the RISK 3 areas were the vector's medium risk occurrence areas. The risk areas showed a trend of patchy dispersion centered on major endemic CLADs and medium-high risk occurrence areas of the vectors. Multi-point re-emergence and local outbreaks appeared in historically endemic areas. Medium-high risks appeared in the suburbs of some large urban regions and their surrounding areas. The distribution of risk areas was concentrated in the Qinling Range in southern Gansu and Shaanxi, the northern Hengduan Range in northern Sichuan, the Lvliang Range and the extended region of Loess Plateau at the cross-border areas of Shaanxi and Shanxi, and hilly areas of the Yanshan-Taihang Range across Shanxi, Henan, and Hebei (Figure 1C).

\section{DISCUSSION}

Between 2015 and 2020, the number of MT-ZVL cases increased significantly in China, and a total of 25 CLADs had re-emerged with 88 cases reported, while the total number of $\mathrm{VL}$ cases declined $(1,3)$. A riskmatrix evaluation on the transmission risks of MTZVL was conducted on account of the classification scores of the endemic CLADs and the occurrence probabilities of $\mathrm{Ph}$. chinensis. Results showed a trend of patchy dissemination centered on major endemic areas and medium-high risk occurrence areas of Ph. chinensis with discrete foci. Multi-point re-emergence and local outbreaks of MT-ZVL are trending in historically endemic areas. A medium-high risk appeared in the suburbs of some large urban regions and their surrounding areas. Timely risk identification and early warning of transmission risks in particular areas lay a

TABLE 1. Risk-matrix evaluation on the transmission risk of mountain-type zoonotic visceral leishmaniasis in 6 endemic PLADs in China, 2015-2020.

\begin{tabular}{ccccc}
\hline $\begin{array}{c}\text { Classification of Occurrence probabilities of } \\
\text { Phlebotomus chinensis }\end{array}$ & \multicolumn{4}{c}{ Classification of MT-ZVL endemic CLADs } \\
\cline { 2 - 6 } & $\begin{array}{c}\text { Class I } \\
\text { (cases } \geq \mathbf{1 0}, 3)\end{array}$ & $\begin{array}{c}\text { Class II } \\
\text { (cases 3-9, 2) }\end{array}$ & $\begin{array}{c}\text { Class III } \\
\text { (cases <3, 1) }\end{array}$ & $\begin{array}{c}\text { Non-endemic } \\
\text { (no case, 0) }\end{array}$ \\
\hline High risk $(0.60-1,3)$ & 6, RISK 1 & 5, RISK 2 & 4, RISK 2 & 3, RISK 3 \\
Medium risk $(0.30-0.59,2)$ & 5, RISK 2 & 4, RISK 2 & 3, RISK 3 & 2, RISK 3 \\
Low risk $(0-0.29,1)$ & 4, RISK 2 & 3, RISK 3 & 2, RISK 3 & 1, RISK 4 \\
\hline
\end{tabular}

Note: MT-ZVL scores were calculated by the summation of the scores of endemic CLADs classification and the scores of occurrence probabilities of Phlebotomus chinensis. The corresponding risk levels were evaluated comprehensively by the scores and opinions of experts.

Abbreviations: MT-ZVL=mountain-type zoonotic visceral leishmaniasis; PLADs=provincial-level administrative divisions; CLADs=countylevel administrative divisions. 
solid foundation for formulating precise prevention and control strategies. Therefore, it is essential to take targeted measures to move the prevention and control barriers forward in medium-high risk areas of MTZVL identified to reduce the transmission risk.

The region covering southern Gansu and northern Sichuan is a traditional MT-ZVL endemic area with 7 super-high-risk areas and 16 high-risk areas. In Shanxi, Shaanxi, Henan, and Hebei, a total of 9 super-highrisk areas and 25 high risk areas emerged. The multipoint re-emergence and local outbreaks of MT-ZVL were significant in historically endemic provinces, especially in Yangquan City (Shanxi Province), Hancheng City (Shaanxi Province), and Anyang City (Henan Province) (1,4-5).

The geographical range of medium-high occurrence risk of $P h$. chinensis was considerably large and likely to expand toward higher latitudes and elevations in the context of global warming (G). The walls of many buildings in rural areas become rough and porous after long-term exposure to sunlight and wind, which provides new breeding grounds for MT-ZVL vectors (7). Due to the weak flying ability of sandflies, the transmission rate of MT-ZVL decreases rapidly based on the distance from the transmission foci (8). The limited range and special ecological habits of $P h$. chinensis were partly responsible for the focal transmission of MT-ZVL. Deeply understanding the environmental influences on the vectors is the key to controlling them.

The rural areas of suburbs and the surrounding areas of several large cities presented as medium-high risk. The number of canines has soared in recent years since they have become the ideal animal for protection and companionship, hindering traditional management methods of mass culling. Investigations found that almost every family in rural areas raised dogs, and the dogs moved without restraint $(4-5,9)$. Once in contact with wild animal sources of infection, MT-ZVL cases could be more prone to appear in rural areas than in cities. Frequent causes of population movement such as tourism and immigration increase imported risk in non-endemic areas. Additionally, the trading of canines has not been supervised by regulatory authorities in a timely manner, leading to greater imported risk (9). Historically, imported dogs in Sichuan have caused reemergent cases (10).

This study was subject to some limitations. The monitoring data of canines was insufficient, and social factors such as population movement and economic conditions were not included in the assessment process.
In the future, it is necessary to carry out more systematic investigations on the vectors and the infectious sources, supplemented by more sensitive detecting methods.

To effectively prevent and control MT-ZVL in China, it is necessary to identify key transmission areas and implement early warnings. In the medium-high risk areas identified in this study, more frequent monitoring of sandflies, canines, and mobile population, establishing joint prevention and control mechanisms, and highlighting health education to the public are recommended.

Conflicts of interest: No conflicts of interest reported.

Acknowledgments: National Institute of Parasitic Diseases, Chinese Center for Diseases Control and Prevention (Chinese Center for Tropical Diseases Research).

Funding: Supported by the Research on Prediction and Early Warning and Intervention Technology of Meteorological Sensitive Parasitic Diseases (No. 2017FY101203), Science and Technology Research Project of the Shanghai Municipal Health Commission (No. 20194Y0359), the Fifth Round of the Three-Year Public Health Action Plan of Shanghai (No. GWV10.1-XK13).

doi: $10.46234 / \mathrm{ccdcw} 2022.029$

\# Corresponding author: Shizhu Li, lisz@chinacdc.cn.

\begin{abstract}
${ }^{1}$ National Institute of Parasitic Diseases, Chinese Center for Disease Control and Prevention (Chinese Center for Tropical Diseases Research); NHC Key Laboratory of Parasite and Vector Biology; WHO Collaborating Centre for Tropical Diseases; National Center for International Research on Tropical Diseases, Shanghai, China; ${ }^{2}$ School of Global Health, Chinese Center for Tropical Diseases Research, Shanghai Jiao Tong University School of Medicine, Shanghai, China.
\end{abstract}

Submitted: December 02, 2021; Accepted: February 21, 2022

\section{REFERENCES}

1. Zhou ZB, Lyu S, Zhang Y, Li YY, Li SZ, Zhou XN. Visceral leishmaniasis-China, 2015-2019. China CDC Wkly 2020;2(33):625 8. http://dx.doi.org/10.46234/ccdcw2020.173.

2. Wang XY, Xue JB, Xia S, Han S, Hu XK, Zhou ZB, et al. Distribution of suitable environments for Phlebotomus chinensis as the vector for mountain-type zoonotic visceral leishmaniasis - six provinces, China. China CDC Wkly 2020;2(42):815 - 9. http://dx.doi.org/10.46234/ ccdcw2020.223.

3. Zhou ZB, Li YY, Zhang Y, Li SZ. Prevalence of visceral leishmaniasis in China during 2015-2018[J]. Chin J Parasitol Parasit Dis 2020;38 (3):339-45. http://www.jsczz.cn/CN/10.12140/j.issn.1000-7423.2020. 03.013. (In Chinese).

4. Wang FP, Chen S, Liu DL, Li GZ, Liu G, Zhang Y, et al. Characteristics and risk factors of the Kala-Azar in Hancheng City, Shaanxi province, China[J]. Chin J Zoon 2018;34(8):756-60. http:// www.rsghb.cn/CN/10.3969/j.issn.1002-2694.2018.00.127. 
(In Chinese).

5. Zheng YH, Bai YF, Tie P, Yan CF, Wang T, Wang JY, et al. Epidemiological characteristics of visceral leishmaniasis in Shanxi province, 2010-2019[J]. Chin J Parasitol Parasit Dis 2021;39 (3):352-8. http://www.jsczz.cn/CN/10.12140/j.issn.1000-7423.2021. 03.009. (In Chinese).

6. Carvalho BM, Rangel EF, Ready PD, Vale MM. Ecological niche modelling predicts southward expansion of Lutzomyia (Nyssomyia) flaviscutellata (Diptera: Psychodidae: Phlebotominae), vector of Leishmania (leishmania) amazonensis in South America, under climate change. PLoS One 2015;10(11):e0143282. http://dx.doi.org/10.1371/ journal.pone.0143282.

7. Gu ZQ, Wu SX, Cui FZ, Jia L, Huang L, Zhang Y. A bionomic investigation and analysis of sandflies, the vectors of Kala-azar, in Sanmenxia, Henan province, China[J]. Chin J Vector Biol Control
2021;32(5):590-3. http://www.bmsw.net.cn/CN/10.11853/j.issn. 1003.8280.2021.05.016. (In Chinese).

8. WHO. Control of the leishmaniases: WHO TRS Nº49. Geneva: World Health Organization. 2010. https://www.who.int/publications/i/ item/WHO-TRS-949. [2021-8-3].

9. Li YX, Cheng R, Zhou RM, Yang CY, Zhang HW, Tian LG, et al. Epidemiological investigation on a new local case of visceral leishmaniasis in Luoyang City, Henan province[J]. Chin J Parasitol Parasit Dis 2021;39(3):410-3. http://www.jsczz.cn/CN/10.12140/j. issn.1000-7423.2021.03.022. (In Chinese).

10. Gao B, Li GR, Wei HY, Wu YX, Zhang WH, Tang ZM, et al. Epidemiological investigation of Kala-azar in Sichuan Province[J]. J Prev Med Inf 2000;16(2):142-3. https://d.wanfangdata.com.cn/ periodical/QK200001273829. (In Chinese). 